J3eA, Journal sur l'enseignement des sciences et technologies de l'information et des systèmes, Volume 4, Hors-Série 1, 10 (2005)

DOI : http://dx.doi.org/10.1051/bib-j3ea:2005610

(C) EDP Sciences, 2005

Composants à semi-conducteur de puissance pour des applications à haute température de fonctionnement

B. Allard ${ }^{1}$, G. Coquery ${ }^{2}$, L. Dupont ${ }^{2,3}$, Z. Khatir $^{2}$, M. Lazar ${ }^{1}$,S. Lefebvre ${ }^{3}$, R. Meuret ${ }^{4}, \mathrm{H}$. Morel ${ }^{1}$ et D. Planson ${ }^{1}$

${ }^{1}$ CEGELY INSA de Lyon, CNRS UMR5005

Bât. Léonard de Vinci, 21 avenue Jean Capelle

F-69621 Villeurbanne CEDEX, France

${ }^{2}$ LTN INRETS, 2 avenue Général Malleret-Joinville, F-94114 Arcueil, France

${ }^{3}$ SATIE, CNAM ENS Cachan, CNRS UMR8029

61 avenue du Président Wilson , F-94235 Cachan CEDEX, France

${ }^{4}$ Hispano-Suiza, Rond Point René Ravaud, Réau

F-77550 Moissy-Cramayel, France 


\title{
Composants à semi-conducteur de puissance pour des applications à haute température de fonctionnement
}

\author{
B.Allard $^{(1)}$ G.Coquery $^{(2)}$ L.Dupont $^{(2,3)}$ Z.Khatir $^{(2)}$ M.Lazar $^{(1)}$ S.Lefebvre $^{(3)}$ R.Meuret $^{(4)}$ H.Morel $^{(1)}$
D.Planson
}

(1) CEGELY INSA de Lyon CNRS UMR5005, Bât. Léonard de Vinci, 21, avenue Jean Capelle 69621 VILLEURBANNE CEDEX

(2) LTN INRETS, 2 av Général Malleret-Joinville 94114 ARCUEIL

(3) SATIE, CNAM ENS Cachan, CNRS UMR8029, 61 Av. du président Wilson 94235 Cachan cedex

(4) Hispano-Suiza, Rond Point René Ravaud - Réau 77550 Moissy-Cramayel

\begin{abstract}
Résumé-L’article décrit les principales limitations (puce, connexions et assemblage) à la montée en température des composants à semi-conducteur de puissance, et montre quels peuvent être en 2003 les composants les mieux adaptés pour des applications à haute température de fonctionnement telles qu'on peut les rencontrer dans le domaine de l'aviation.
\end{abstract}

\section{INTRODUCTION}

Dans le domaine de l'aviation, la généralisation en cours ou souhaitée de l'utilisation d'actionneurs électriques oblige à une utilisation toujours plus importante de l'électronique de puissance. Cette utilisation croissante de l'électricité dans ce domaine d'application est motivée notamment par une réduction de la masse de l'appareil, de la consommation d'énergie et de l'émission de gaz polluants. Dans le cas de l'avion “plus électrique ”, l’introduction et la généralisation des actionneurs électriques doit permettre, à terme, une réduction de $10 \%$ du coût d'investissement et de maintenance ainsi que $20 \%$ sur la masse de l'appareil.

On cherche généralement à placer les convertisseurs d'électronique de puissance au plus près des actionneurs qu'ils servent à piloter, ce qui peut être à proximité du réacteur dans un avion. Dans ce cas particulier, la température ambiante d'utilisation des composants est particulièrement élevée $\left(200^{\circ} \mathrm{C}\right.$ environ au voisinage du réacteur en vol). Le régime de fonctionnement établi est excessivement contraignant, mais il faut également prendre en compte les phases transitoires (décollage et atterrissage) pendant lesquelles la température du réacteur peut varier dans le pire des cas de $-55^{\circ} \mathrm{C}$ (hiver, régions polaires) à + $200^{\circ} \mathrm{C}$ (régime établi en vol), avec des vitesses de variation de la température du réacteur de l'ordre de $10^{\circ} \mathrm{C} / \mathrm{min}$. Il faut rajouter à ces contraintes thermiques extrêmement sévères celle de la durée de vie souhaitée qui est de l'ordre de 50.000 heures pour les équipements, ce qui correspond à 5000 phases de décollage/atterrissage pour des durées moyennes de vol de l'ordre de 10 heures.
Cet article fait suite à une collaboration engagée entre Hispano-Suiza, le SATIE (ENS Cachan, CNAM), le LTN de l'INRETS et le CEGELY(INSA de Lyon). L'objectif de cette collaboration est la réalisation d'un convertisseur (onduleur triphasé) pour la commande d'un EMA (Electro Mechanical Actuator) de puissance inférieure à 5kW.

Les composants et modules à semi-conducteur de puissance sont basés sur des technologies $\mathrm{Si}$, SOI ou SiC dont les parties actives sont montées sur un substrat céramique et connectées entre elles, généralement par des fils de bonding. Le substrat céramique est brasé sur la semelle. La température élevée de fonctionnement et les variations de température imposées lors des phases de décollage/atterrissage soulèvent principalement le problème de la durée de vie et de la fiabilité de ces composants.

Bien qu'il existe déjà des puces spécifiquement développées pour fonctionner à $175{ }^{\circ} \mathrm{C}$ (dans le domaine automobile notamment), il n'existe pas encore aujourd'hui de composants commercialisés spécifiés pour les contraintes thermiques rencontrées dans le domaine de l'aviation. Seules de très rares puces pouvant fonctionner à haute température sont disponibles ou en cours de développement (Transistors MOS sur SOI, transistors bipolaires, Diodes Schottky SiC, JFET SiC). Le fabricant Honeywell propose par exemple un transistor MOS sur SOI (HTANFET $90 \mathrm{~V}-0.4 \Omega$ à $25^{\circ} \mathrm{C}$ ) spécifié à $225^{\circ} \mathrm{C}$. Toutefois, tous ces composants n'ont pas encore été caractérisés à ces températures extrêmes et leur comportement électrique (chute de tension directe, vitesse de commutation, robustesse) reste mal connu. Le principal problème reste toutefois la tenue à ces températures et aux cycles de variation de la température de l'assemblage mécanique de la puce sur le substrat, du substrat sur le socle et des connexions entre le puce et les broches extérieures.

Les risques de vieillissement et de dysfonctionnement sont multiples et peuvent apparaître à plusieurs niveaux :

Les puces semi-conductrices : la température élevée de fonctionnement accélère le vieillissement de la puce (dégradation de l'oxyde de grille par injection de porteurs 
chauds pour les composants à grille isolée, problèmes de corrosion, de dégradation des métallisations par électromigration ...). Des essais spécifiques doivent être mis en œuvre pour mettre en évidence les modes de défaillance et garantir la durée de vie de la puce.

L'assemblage : L'assemblage de la puce sur le substrat et du substrat sur le socle ou boîtier par l'intermédiaire de brasures est une des principales faiblesses des composants à semi-conducteur de puissance. La différence entre les coefficients de dilatation thermique (CTE) des différents éléments de l'assemblage (puce Si ou SiC, substrat céramique, socle) sont à l'origine de contraintes mécaniques au sein des brasures. Les cycles de température imposés lors des phases de décollage/atterrissage peuvent entraîner le délaminage des brasures et diminuer fortement la durée de vie des composants. D’autre part, la défaillance peut provenir des fils de bonding qui assurent les connexions des différentes puces entre elles ou les connexions vers l'extérieur. Les soudures des fils de bonding, placées sur la surface de la puce voient les variations de température ambiante ajoutées aux excursions de température imposées par la dissipation de puissance dans la puce (pertes). Les contraintes mécaniques qui en découlent peuvent être à l'origine de la levée des fils de bonding.

En s’appuyant sur la bibliographie et des résultats expérimentaux réalisés dans nos laboratoires, nous décrirons dans cet article l'état de l'art et les développement prévus des composants à semi-conducteur de puissance pour une électronique de puissance haute température. L'automobile étant un secteur moteur dans ce domaine, une partie de l'article sera consacrée aux avancées récentes qui ont été portées par ce secteur d'activité.

\section{Problematique des Composants A SEMI- CONDUCTEUR DE PUISSANCE A HAUTE TEMPERATURE}

A. Au niveau de la puce active

a) Tenue en tension et polarisation directe.

La figure 1 décrit une jonction $\mathrm{P}^{+} \mathrm{NN}^{+}$typique d'un composant bipolaire de puissance en polarisation inverse.

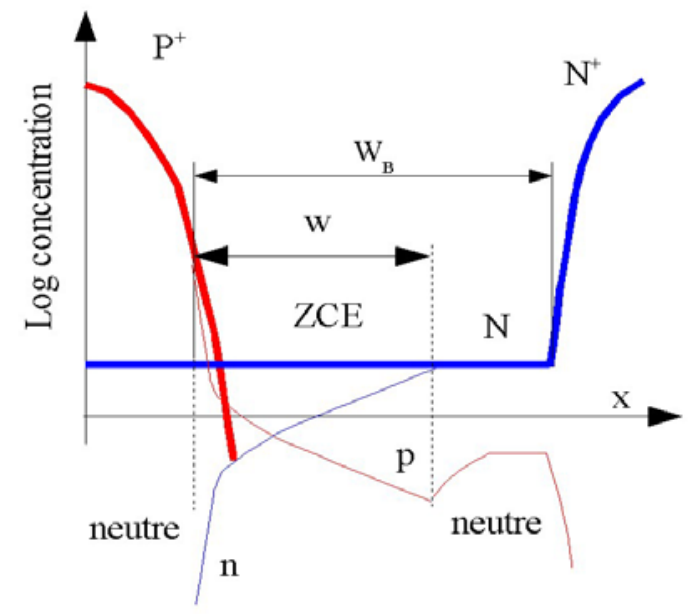

Fig.1: Représentation typique d'une zone de charge d'espace d'une diode $\mathrm{P}^{+} \mathrm{NN}^{+}$en polarisation inverse

Les figures 2 et 3 donnent la tenue en tension en fonction du dopage $\mathrm{N}_{\mathrm{D}}$ et l'étendue de la couche faiblement dopée $\mathrm{W}_{\mathrm{B}}$ pour du silicium et du SiC. Les régions entourées correspondent à une chute de tension directe optimisée.

Pour une tenue en tension donnée, $\mathrm{V}_{\mathrm{BR}}$, la chute de tension à l'état passant est optimale dans la région coudée. Aussi pour une jonction optimisée la tenue en tension diminue quand le dopage augmente. De plus, un composant unipolaire en $\mathrm{SiC}$ a une chute de tension plus faible que le composant Si de même calibre en tension, à cause du dopage plus élevé.

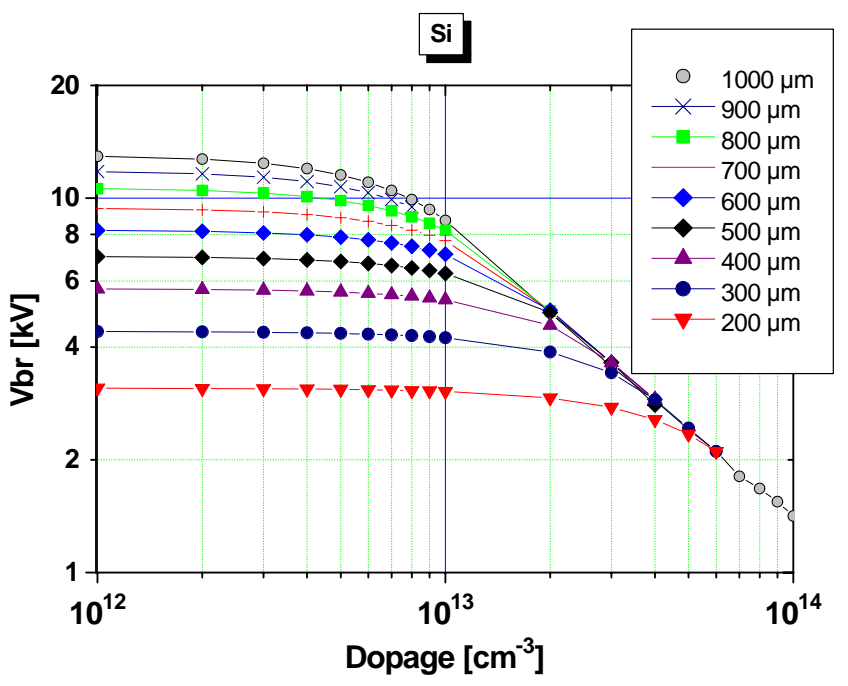

Fig. 2: tenue en tension d'une jonction PIN en Si en fonction du dopage $N_{D}$ et de $\mathrm{W}_{\mathrm{B}}$.

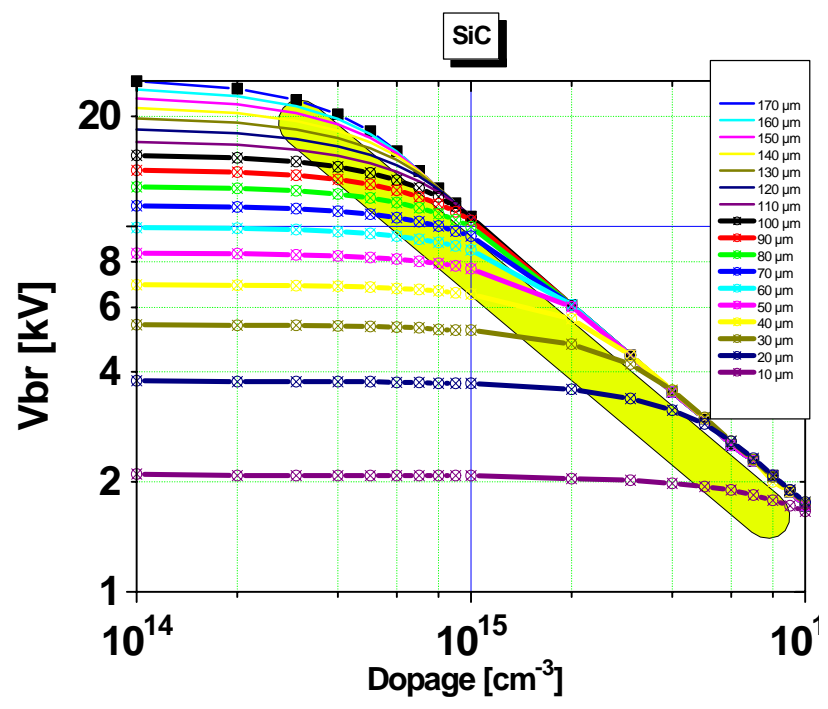

Fig. 3: tenue en tension d'une jonction PIN en SiC en fonction du dopage $N_{D}$ et de $\mathrm{W}_{\mathrm{B}}$.

\section{b) courant de fuite d'une jonction $P N$}

La température modifie de façon significative les paramètres physiques du cristal semi-conducteur. En particulier, l'élévation de la température entraîne une augmentation considérable de la concentration intrinsèque $\left(n_{i} \propto T^{3 / 2} \exp (-E g / 2 k T)\right)$.

Le problème principal survient alors lorsque la concentration intrinsèque n'est plus négligeable devant la concentration d'impuretés de la région faiblement dopée de base qui assure la tenue en tension; les types de dopages ne 
sont alors plus affirmés et le composant perd toute capacité de tenue en tension.

C'est la raison pour laquelle les composants basse tension, avec un dopage de la région de base plus élevée sont a priori mieux adaptés aux applications à température de fonctionnement très élevée. C’est également la raison pour laquelle les composants à grand gap sont mieux adaptés pour des applications à température de fonctionnement élevée (Fig.4). Pour des dispositifs haute tension (1000V), la limite théorique du silicium est de $150^{\circ} \mathrm{C}$ alors quelle est de $900^{\circ} \mathrm{C}$ pour du $6 \mathrm{H}-\mathrm{SiC}$ [1]. Pour des dispositifs $100 \mathrm{~V}$, celle-ci est de l'ordre de $250^{\circ} \mathrm{C}$ pour du Si (Fig.5).

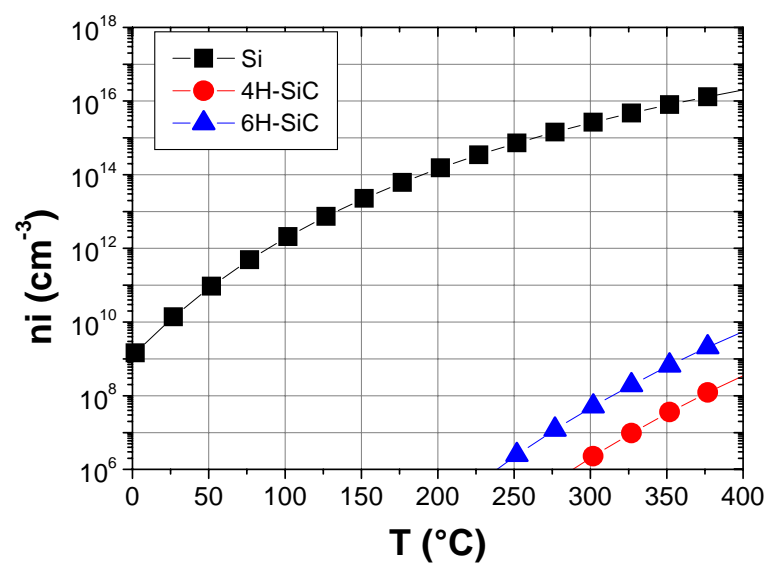

Fig. 4: concentration intrinsèque fonction de la température

La seconde conséquence de l'augmentation de $\mathrm{n}_{\mathrm{i}}$ concerne l'accroissement du courant de fuite en volume des jonctions PN qui est considéré comme une des principale limitation à l'augmentation de la température [1,2]. Ce courant de fuite a deux origines. Une composante due à la génération thermique dans la zone de charge d'espace qui varie proportionnellement à ni. La seconde composante est celle du courant de diffusion qui varie avec $\mathrm{ni}^{2}$. Les coefficients de proportionnalité sont tels que, aux températures usuelles, le courant de diffusion est généralement négligeable devant le courant de génération thermique. Ce dernier ne devenant prépondérant qu’à haute température à cause de la valeur de $\mathrm{ni}^{2}$. Remarque: la diffusion et la génération n'ont pas lieu aux mêmes endroits!

La figure 5 montre à titre indicatif les températures maximales d'utilisation de différents matériaux semiconducteur lorsque le courant de génération thermique est pris comme limite à la montée en température.

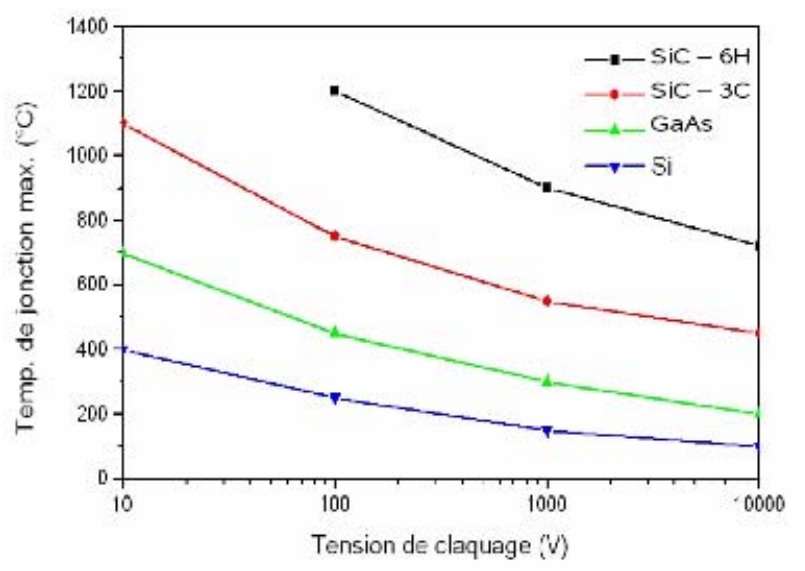

Fig. 5 : Température max. fonction de la tension de claquage pour différents matériaux [1]
En dehors des problèmes de tenue en tension et de courant de fuite, ce sont ceux liés à la tenue des oxydes de grilles avec la température et sous fort champ électrique $\left(\mathrm{T}_{\mathrm{DDB}}\right)$ qui limitent la durée de vie des dispositifs à grille isolée [3]. De manière corollaire, les effets d'injection de porteurs chauds dans les oxydes est aussi une limitation à l'utilisation de ces composants à haute température [4].

\section{c) Courant de fuite d'une diode Schottky}

Une diode Schottky est un contact redresseur métalsemiconducteur. Les mécanismes de transports sont assez complexes et encore assez mal modélisés. Ils incluent l'émission thermoionique (comme dans les diodes à lampes), la génération et la diffusion (comme ci-dessus), la réduction de la barrière (force image) et l'effet tunnel (sur des distances très courtes où la plupart des théories ne sont plus valables). Résultat, la littérature dispose de nombreux modèles en général pas totalement satisfaisants. Toutefois les courants de fuites qui existent dans une jonction PN existent aussi dans une diode Schottky. En conséquence les courants de fuites sont toujours plus grands dans une diode Schottky que dans une diode bipolaire de même calibre $\left(\mathrm{N}_{\mathrm{B}}, \mathrm{W}_{\mathrm{B}}\right.$, Fig.2 et Fig.3). De plus la dépendance en fonction de la température se fait au détriment de la diode Schottky qui a toujours une croissance du courant de fuite en fonction de la température plus grande que la jonction PN de même géométrie.

\section{d) Spécificité du SOI vis à vis des courants de fuite}

En technologie SOI (Fig. 6), il apparaît deux types de transistors, en fonction de l'épaisseur de la couche semiconductrice active déposée sur l'oxyde profond. Si cette épaisseur est suffisamment grande, il existe toujours une zone en condition de quasi-neutralité, (Fig. 7) sous la zone de canal, en polarisation normale, ou bien sous une zone de charge d'espace lors du blocage.

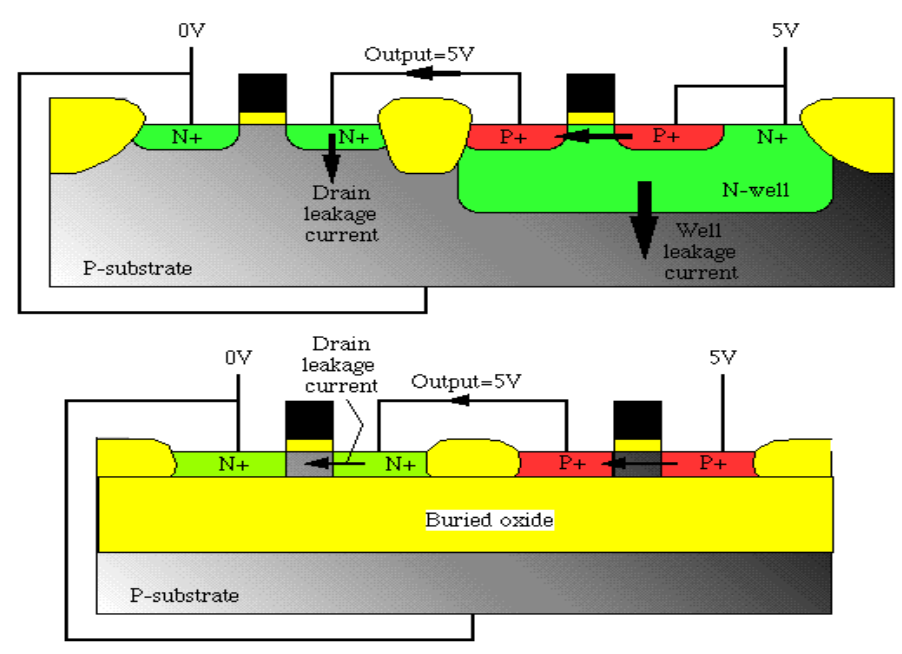

Fig. 6: Technologie CMOS "bulk" et SOI 


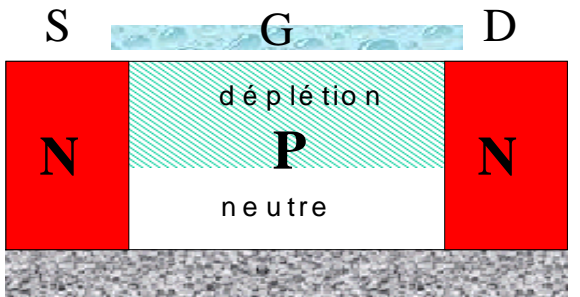

Trans is tor MOS SOI, partielle m e nt d ép lé té.

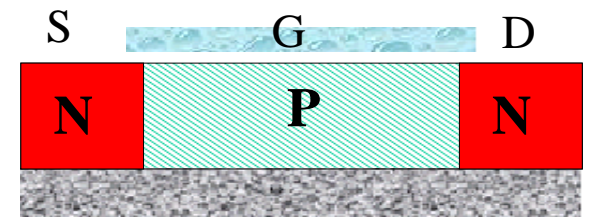

Trans is tor MOS SOI, com plè te m e nt d é plé té.

Fig. 7: différence entre les transistors partiellement et complètement déplétés

Dans ces conditions le courant de fuite d'un transistor MOSFET est surtout lié à la contribution de diffusion dans la zone quasi-neutre, d'autant plus que la température est élevée. On parle de structure SOI " partiellement déplétée ». (Fig. 7).

Quand l'épaisseur de la couche active au-dessus de l'oxyde, est de faible épaisseur, le canal ou la zone de charge d'espace occupent tout le volume de la couche active. Il s'agit d'une structure SOI dite " complètement déplétée ». Dans ce cas, seule la génération contribue au courant de fuite.

\section{B. Au niveau de l'assemblage}

La figure suivante montre les couches et matériaux entrant dans la constitution d'un assemblage de puissance.

puce

semi-conductrice

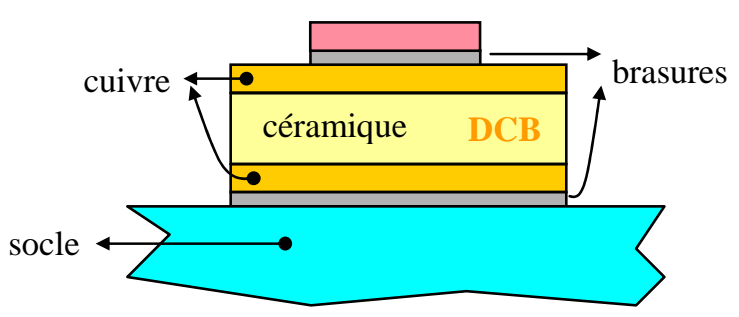

Fig. 8 : assemblage d'une puce sur DCB

\begin{tabular}{|c|c|c|c|}
\cline { 2 - 4 } \multicolumn{1}{c|}{} & Matériau & $\begin{array}{c}\mathrm{CTE} \\
(\mathrm{ppm} / \mathrm{K})\end{array}$ & $\begin{array}{c}\lambda \\
(\mathrm{W} / \mathrm{mK})\end{array}$ \\
\hline \multirow{2}{*}{ Puce } & Silicium & 2.6 & 150 \\
\cline { 2 - 4 } & $\mathrm{SiC}$ & 3.7 & 390 \\
\hline \multirow{2}{*}{ Brasure } & \multicolumn{2}{|c|}{} \\
\hline \multirow{2}{*}{ Céramique } & $\mathrm{AlN}$ & 3.1 & 190 \\
\cline { 2 - 4 } & $\mathrm{Al}_{2} \mathrm{O}_{3}$ & 5.5 & 30 \\
\hline Brasure & \multicolumn{3}{|c}{} \\
\hline \multirow{2}{*}{ Socle } & $\mathrm{Cu}$ & 16 & 390 \\
\cline { 2 - 4 } & $\mathrm{AlSiC}$ & $6.5-12$ & $180-220$ \\
\hline
\end{tabular}

TABLEAU 1 : PROPRIETES DES PRINCIPAUX MATERIAUX CONSTITUANT UN ASSEMBLAGE DE PUISSANCE [5]

Sous des contraintes de cyclage en température, le délaminage apparaît plus rapidement dans la brasure de plus grande dimension, à l'interface entre le substrat céramique et le socle. Pour chercher à réduire la différence de CTE entre la céramique et le socle plusieurs types d'assemblage peuvent être envisagés.

Céramique Al2O3 / Socle Cuivre : Solution économique utilisée dans l'automobile. Toutefois, la mauvaise conductivité thermique de l'alumine pénalise ce type d'assemblage (résistance thermique élevée)

Céramique AIN / Socle AISiC: Malgré le fait que la conductivité thermique de l'AlSiC est plus faible que celle du cuivre, la bonne conductivité thermique de l'AlN confère à cet assemblage une meilleure résistance thermique que le précédent. D'autre part, l'utilisation d'un socle MMC (Metal Matrix Composite) AlSiC permet d'obtenir une bonne robustesse de l'assemblage aux cycles de variation de température [6]

\section{Au niveau des connexions}

Pour la plupart des composants de puissance, les connexions entre les puces et le boîtier s'effectuent par l’intermédiaire de fils de bonding (Fig.9).

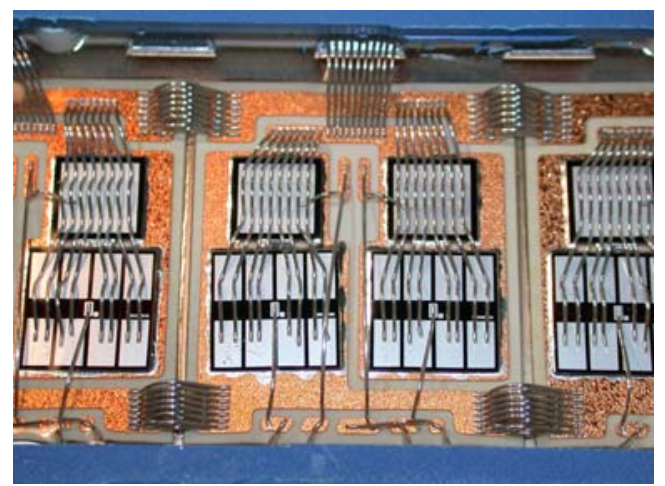

Fig.9 : Module EconoPack Eupec 600V 200A, connexions d'IGBT et de diodes anti-parallèle par fils de bonding

Le diamètre des fils de bonding est généralement de $300 \mu \mathrm{m}$ dans les modules de moyenne puissance. Une étude récente sur la fatigue thermo-mécanique de modules de puissance dédiés aux applications de traction automobile (véhicule hybride et alterno démarreur) a montré que la levée des fils de bonding pouvait être la principale cause de défaillance [7] lors d'un fonctionnement à haute température (la température de la puce varie entre $85^{\circ} \mathrm{C}$ et $150^{\circ} \mathrm{C}$ dans le cas présent, et celle du boîtier entre $50^{\circ} \mathrm{C}$ et $115^{\circ} \mathrm{C}$ ).

Les applications à haute température peuvent affaiblir à la fois le fil de bonding et le contact qu'il assure sur la métallisation de la puce $[2,8]$. Pour le premier, cela est du à un recuit subit par le fil qui induit des modifications des propriétés mécaniques en réduisant sa résistance à la fatigue ; en ce qui concerne le contact, les réactions qui s'y s'effectuent sont suivies de la formation de composés intermétalliques qui peuvent avoir pour effet de fragiliser mécaniquement le contact et mener à la "levée" des fils ou bien de maintenir le contact en augmentant la résistance électrique (ce qui se traduira également par une levée de fils) [8]. Par ailleurs, nous pouvons signaler que des tests de durée de vie et de fiabilité spécifiques des fils de bonding à haute température (jusqu'à $200^{\circ} \mathrm{C}$ ) ont été menés [9] où non seulement les effets de la température mais aussi ceux des gradients 
de température, des variations $\Delta \mathrm{T}$ ainsi que des vitesse de variation (dT/dt) et des niveaux de courants ont été étudiés.

\section{COMPOSANTS ET MATERIAUX ADAPTES A DES APPLICATIONS A HAUTE TEMPERATURE}

\section{A. Composants silicium}

Seuls des composants basse tension $\left(\mathrm{V}_{\mathrm{BR}}<200 \mathrm{~V}\right)$ sont, a priori, utilisables à température de fonctionnement élevée (supérieure à $200^{\circ} \mathrm{C}$ ). Le CoolMOS, et de façon plus générale les composants à base de super-jonction, peuvent faire exception, dans la mesure où leur région de base (qui assure la tenue en tension) est plus fortement dopée qu'un composant standard de même tenue en tension. A titre indicatif, la figure suivante montre l'évolution des courants de fuite de différents transistors silicium avec la température :
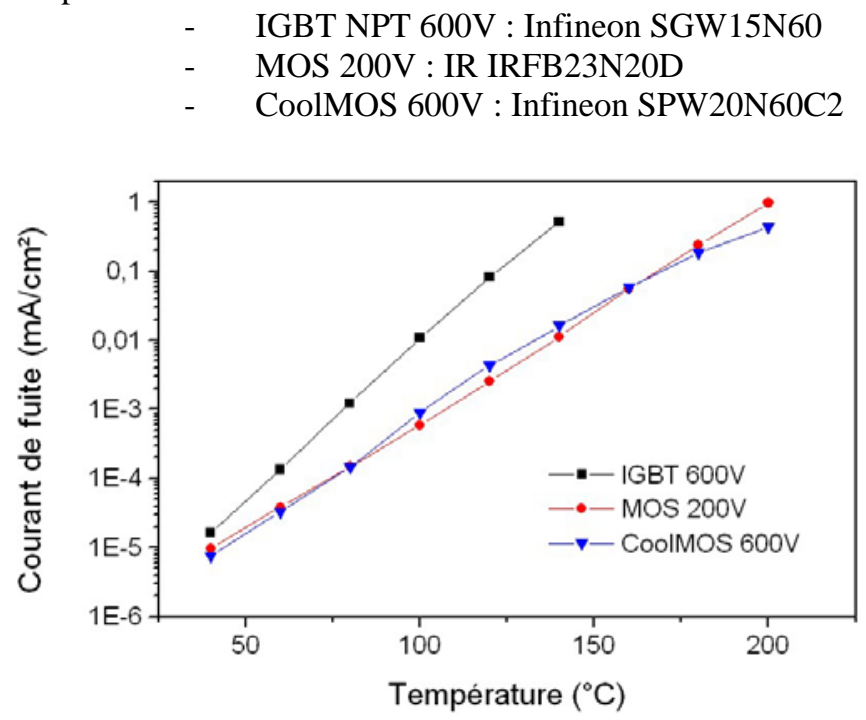

Fig. 10 : Evolution du courant de fuite de différents transistors MOS testés à leur tension de claquage

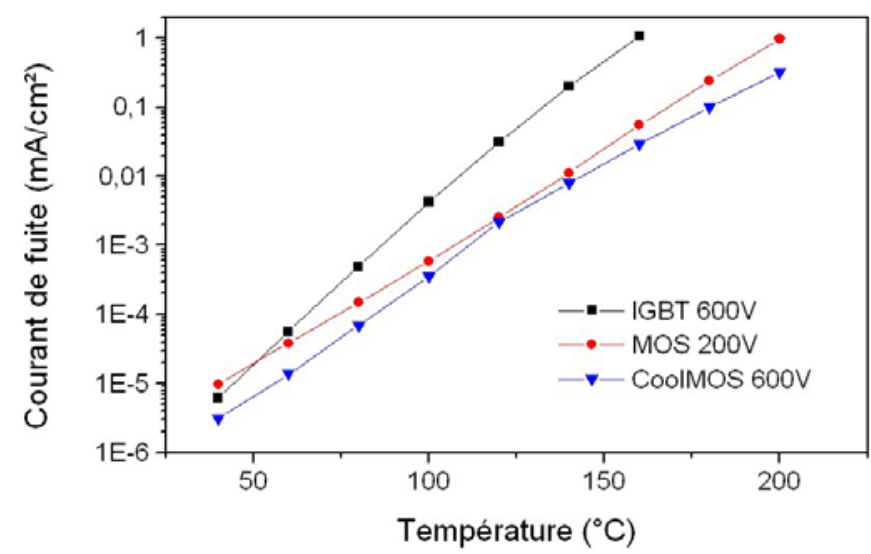

Fig. 11 : Evolution du courant de fuite de différents transistors MOS testés a $\mathrm{V}_{\mathrm{DS}}=200 \mathrm{~V}$

Ces relevés expérimentaux tendent à montrer que la variation du courant de fuite avec la température d'un transistor CoolMOS 600V est très proche de celle d'un transistor MOS 200V. Dans ces conditions, la température maximale d'utilisation d'un CoolMOS $600 \mathrm{~V}$ peut être envisagée voisine de celle d'un MOS 200V.

\section{B. SOI}

Les composants développés sur SOI (Silicon on Insulator) présentent une surface active plus faible que celle de composants «bulk» de caractéristiques similaires. En effet la couche active est entourée de murs d'oxyde latéraux qui limitent de fait la surface du composant. Il faut d'autre part considérer les structures dites " partiellement déplétée » ou « complètement déplétée » $[10,11]$. Les composants « complètement déplétés » présentent les meilleures caractéristiques de courant de fuite avec la température, comme le précise la figure 12 suivante.

Par ailleurs la présence d'une couche d'oxyde enterrée en SOI empêche l'accumulation de charges comme celles qui peuvent polluées le «bulk » en technologie classique.

Dans le cas d'une technologie CMOS, le bulk agit comme concentrateur de courant de fuite, et pose un problème plus ardu pour le concepteur de circuit, qui n'a qu'à se soucier d'un seul chemin de courant de fuite en SOI [12].

A contrario, dans une structure SOI «partiellement déplétée ", les charges injectées dans la zone quasi-neutre modifient la tension de seuil dans le sens défavorable, comme dans le cas d'une technologie bulk.

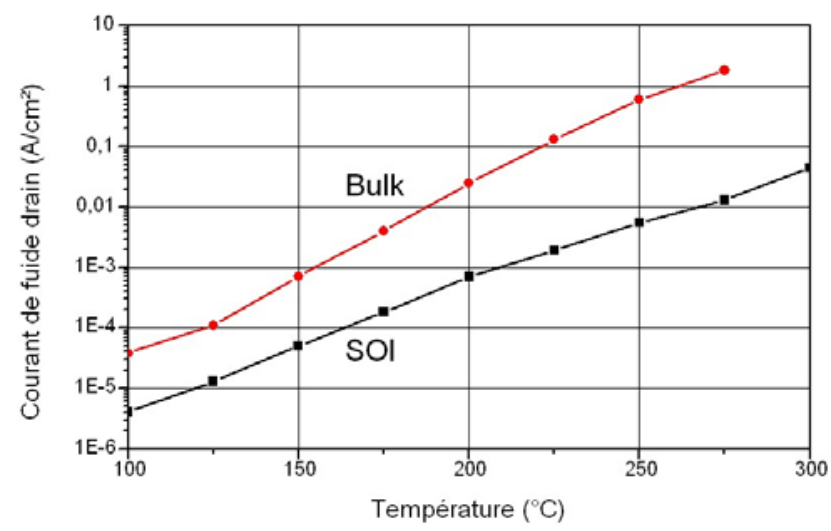

Fig. 12: Comparaison d'un transistor Bulk avec un transistor sur SOI [1]

\section{IDSS vS TEMPERATURE}

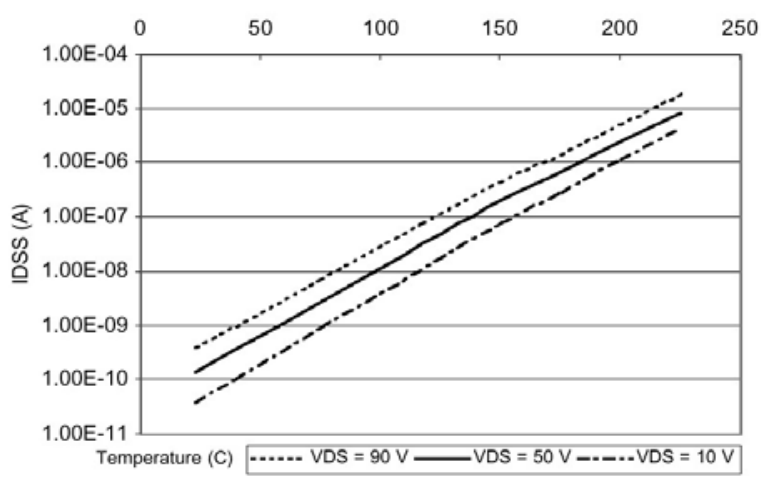

Fig. 13: Courant de fuite fonction de la tension et de la température d'un transistor de puissance sur SOI (HTANFET, Honeywell) 


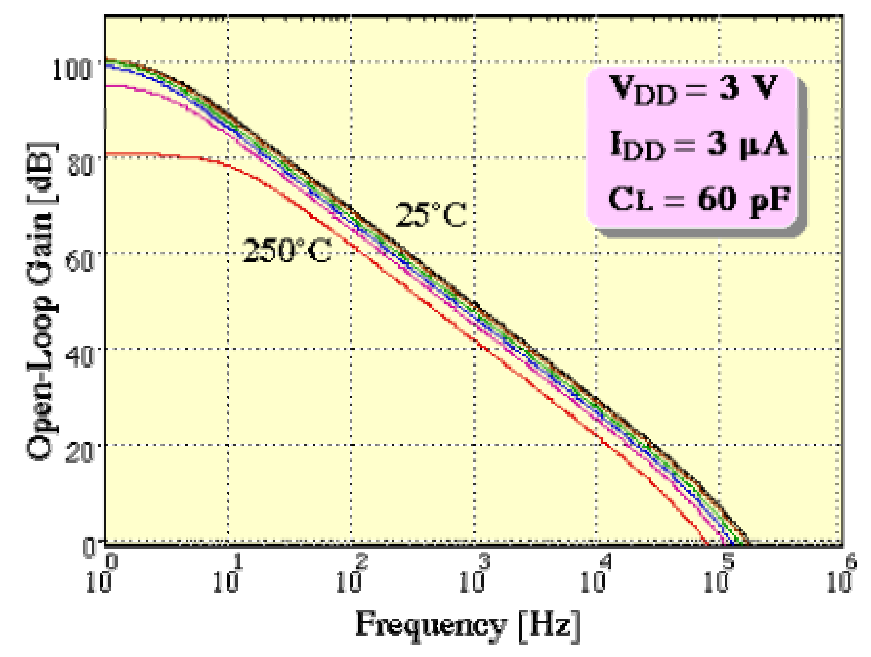

Fig. 14: Gain en boucle ouverte d'un amplificateur opérationnel de type Miller, en SOI-CMOS [13]

Les caractéristiques et les performances des technologies sur SOI sont démontrées, et constituent une large part des discussions de la conférence HiTEC (International HighTemperature Electronics Conference). Par ailleurs de nombreuses structures analogiques et numériques ont été réalisées en CMOS sur SOI, et leurs performances intrinsèques à haute température démontrent la faisabilité d'une telle technologie. Par contre seules des puces semiconductrices ont été testées, et il reste à démontrer le comportement au sein d'un assemblage.

Enfin, l'AlN constitue sans doute une approche plus intéressante que le $\mathrm{SiO}_{2}$ pour l'oxyde enterré, dans le cas de composant à fort courant. Le gain se situe au niveau de la conductibilité thermique, qui représente un paramètre important dans le cas de fonctionnement en ambiante à forte température.

\section{Composants SiC}

Les seuls composants disponibles facilement en 2003 sont des diodes Schottky 300V et 600V. La Fig. 15 montre à titre indicatif la variation du courant de fuite d'une diode Schottky SiC $600 \mathrm{~V}$ en fonction de la température, pour différents tensions appliquées à ses bornes.

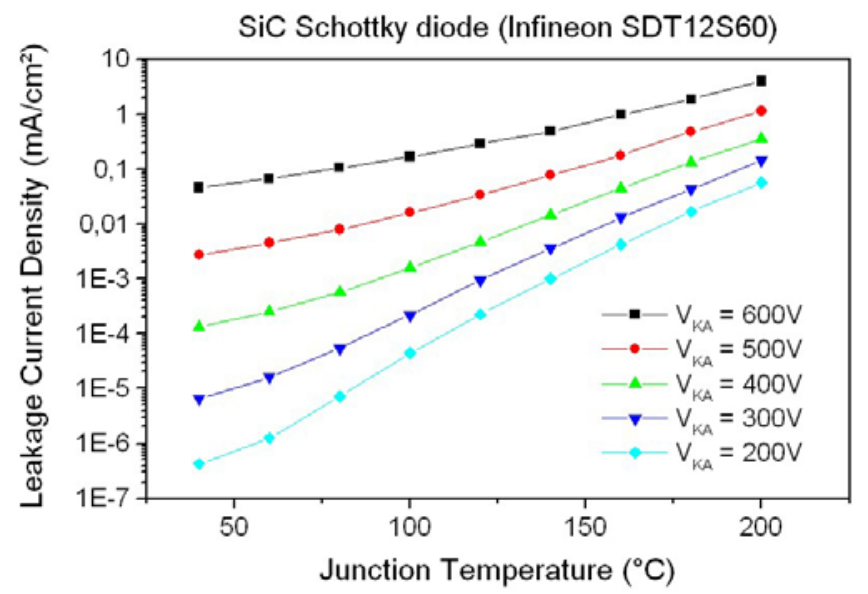

Fig. 15 : Courant de fuite d'une diode Schottky SiC fonction de la température pour différentes tensions de polarisation

La figure suivante compare les densités de courants de fuite d'une diode Schottky $\mathrm{SiC} 600 \mathrm{~V}$ à une diode PIN
Silicium $600 \mathrm{~V}$ spécifiée à $175^{\circ} \mathrm{C}$ de température maximale de jonction. Les résultats sont présentés pour une tension de 400V appliquée aux bornes de la diode.

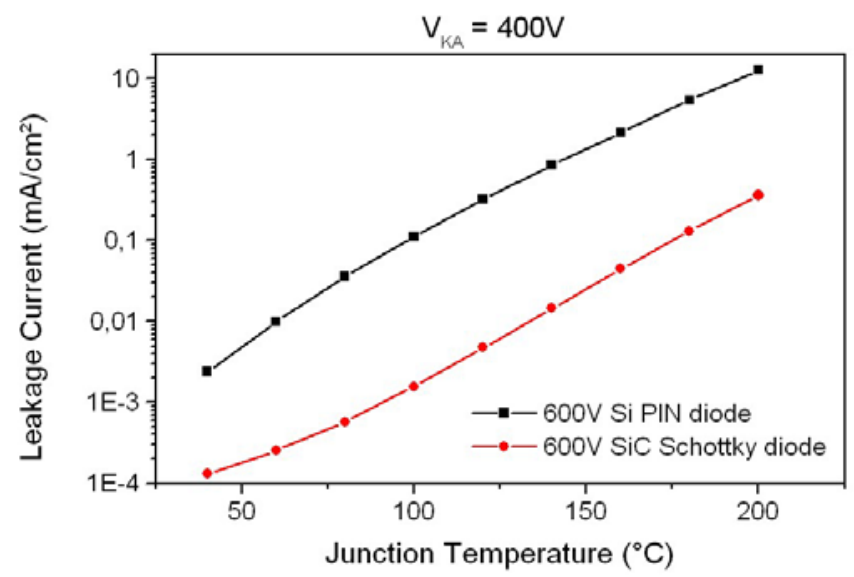

Fig. 16 : Comparaison de diodes 600V, Schottky SiC et PIN Silicium

Cette figure montre clairement l'intérêt de la diode Schottky SiC par rapport à une diode silicium PIN de même tenue en tension.

\section{APPLICATIONS AUTOMOBILES}

A bord des véhicules, en particulier haut de gamme, apparaissent de plus en plus d'organes électriques pour répondre à des besoins de confort : vitres électriques, sièges électriques, climatisation, frein à main, multimédia, direction assistée électrique, ventilation électrique, boîtes de vitesse robotisée et automatique, commande d'arbres à cames, freinage électro-hydraulique, suspensions, aide à la conduite... On estime qu'à l'horizon 2010, le besoin électrique à bord sera de l'ordre de 5 à $10 \mathrm{~kW}$ et il devient nécessaire de monter le niveau de tension du réseau à $42 \mathrm{~V}$ avec les batteries, les convertisseurs de tension et les générateurs de tension correspondants [13, 14]. C'est le cas par exemple de l'onduleur intégré à l'alterno-démarreur (ISG - Integrated Starter Generator) d'une puissance nominale de $6 \mathrm{~kW}$ pour lequel le marché prévu pour l'Europe à l'horizon 2010 est d'environ 1Mpièces/an.

Par ailleurs, un second niveau de puissance, de l'ordre de $25 \mathrm{~kW}$, est requis pour des fonctions traction de moteur électrique pour les véhicules hybrides. Le réseau électrique correspondant est alors indépendant avec un niveau de tension nominale de $400 \mathrm{~V}$.

Ces deux réseaux nécessitent des dispositifs électroniques de puissance qui satisfassent à la fois des contraintes de coût et un besoin en terme de durée de vie de l'ordre de $8000 \mathrm{~h}$ de fonctionnement, (ce qui correspond à environ $200.000 \mathrm{~km}$ ) dans un environnement thermique nominal de $90^{\circ} \mathrm{C}$ pouvant monter jusqu'à $120^{\circ} \mathrm{C}$ et de l'ordre de 400 kcycles de puissance [7].

En ce qui concerne le réseau $42 \mathrm{~V}$ les dispositifs développés sont principalement des dispositifs à MOSFETS 70V-90V à technologie Trench [17, 18]. Cette technologie permet de réduire la longueur des lignes de courant dans l'axe vertical drain-source grâce aux canaux MOSFETs verticaux contrairement aux structures classiques. La réduction de la résistance à l'état passant $\left(\mathrm{R}_{\mathrm{DSON}}\right)$, qui peut être significative pour les dispositifs de tenue en tension de 100 à $200 \mathrm{~V}$, permet d'augmenter légèrement les densités de 
courants et de réduire les tailles des puces. IXYS propose déjà dans son catalogue ce type de dispositif (VWM3500075P) qui est un module onduleur triphasé intégré (sixpack) à MOSFET Trench (Fig.17) spécifié à $175^{\circ} \mathrm{C}$.

Des dispositifs de ce type ont également été fabriqués par Infineon (Fig.18) et ont été testés en terme de fiabilité en environnement haute température dans le cadre du programme HIMRATE [7].

En ce qui concerne les applications hybrides, des modules onduleurs tout intégrés à IGBT sont nécessaires en calibre 600V-200A. Pour cette application, le courant moyen est de 50A avec 180A crête sous environ $400 \mathrm{~V}$ de tension nominale. Pour les deux réseaux, il apparaît que l'élément le plus fragile à l'environnement thermique et aux contraintes de cyclages reste l'assemblage. En particulier, les fils de bondings qui ont tendance à se soulever et la délamination de la brasure entre le DCB et la semelle. Pour les deux types de modules, MOSFETs et IGBT, des efforts ont été faits notamment en terme de techniques de refroidissement, afin de refroidir directement la semelle des modules par contact direct du liquide en circulation.

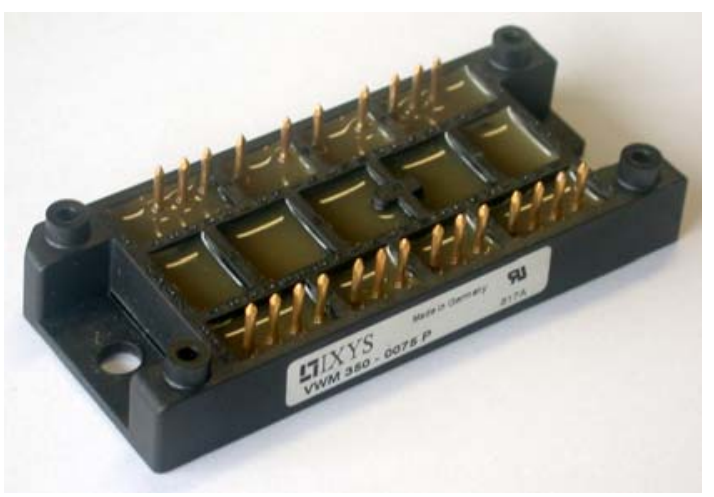

Fig.17 Module IXYS (VWM350-0075P)

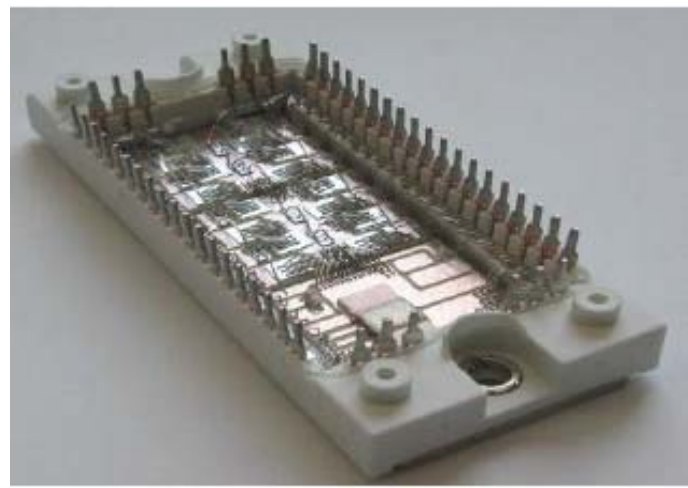

Fig.18 Module MOSFET ([7] source Eupec)

\section{CONCLUSIONS}

La bonne connaissance de la physique des composants à semiconducteur, permet une meilleure optimisation des composants pour leur utilisation à haute température. Par exemple, le cool-MOS et les composants SOI en sont de bonnes illustrations. Par ailleurs, les propriétés intrinsèques des matériaux semiconducteurs (gap, tenue en tension) conduisent à l'utilisation de matériaux plus performants comme le SiC. La diode Schottky SiC est déjà commercialement disponible. Toutefois des diodes bipolaires SiC présenteront des caractéristiques plus intéressantes encore à haute température de fonctionnement.

Enfin, la problématique de l'assemblage et de la connectique demeure un point clef dont la nature reste la même pour les applications avec des puces en $\mathrm{Si}$ ou en $\mathrm{SiC}$, avec toutefois une excursion en température plus importante dans le cas des puces SiC.

Finalement, nous devons nous attendre à repousser sensiblement les limites d'utilisation à haute température des composants de puissance.

\section{BIBLIOGRAPHIE}

[1] W. Wondrak, . "Physical limits and lifetime limitations of semiconductor devices at high temperatures”, Microelectronics Reliability $\mathrm{N}^{\circ} 39$, 1999, pp 1113-1120

[2] P. McCluskey \& al. « Packaging of power electronics for high temperature applications » Advancing Microelectronics, January/February 1998, pp 19-24

[3] J. Manca \& al. "Reliability aspects of high temperature power MOSFETS”, Microelectronics reliabitlity, № 40, 2000, pp 1679-1682

[4] W. Kanert \& al. "reliability aspects of semiconductor devices in high temperature applications”, ESREF 2003

[5] A.B. Lostetter, F. Barlow, A. Elshabini, "An overview to integrated power module design for high power electronics package”, Microelectronics reliability $40 \mathrm{~J}$.

[6] Coquery, G. and al. "Reliability improvement on the soldering thermal fatigue with AlSiC technology on traction high power IGBT modules, EPE’99, 1999

[7] Coquery \& al. "high temperature reliability on automotive power modules verified by power cycling tests up to $150^{\circ} \mathrm{C}$ ”, ESREF 2003

[8] G.G Harman, "Metallurgical Bonding Systems for high temperature electronics", High-Temperature Electronics, Ed. Randall Kirschman, IEEE Press, 1998.

[9] Passagrilli et all., "Reliability of Au/Al bonding in plastic packages for high temperature $\left(200^{\circ} \mathrm{C}\right)$ and high current applications", Microelectronics Reliability, vol.42, pp.1523-1528, 2002.

[10] Flandre D. et al, « The art of high-temperature FD-SOI CMOS », 3rd European Conference on High-Temperature Electronics, 1999

[11] Flandre D, « High-Temperature Electronics », IEEE Press, New-York, 1998

[12] Colinge J.P. « Silicon-On-Insulator Technology », Kluwer Academic, Amsterdam, 1997

[13] Natarajan S. « SOI design: analog, memory and digital techniques », Kluwer Academic, Amsterdam, 2001

[14] J.P. Eggermont, B. Gentinne, D. Flandre, P.G.A. Jespers, J.P. Colinge, "SOI CMOS operational amplifier for applications up to $300^{\circ} \mathrm{C}$ ", HiTEC 1994.

[15] Pascal BERNARD, Jarvis CARTER et Randy FRANK, “THE 42V CAR OF THE FUTURE” note technique ON-semiconductor.

[16] J.G. Kassakian et D.J. Perreault, "The future of electronics in automobiles”, ISPSD’001, pp.15-19, Osaka, 2001

[17] A. Lindemann, "New Trench Power Mosfets in isolated packages" Note technique IXYS

[18] K. Shenai, "Optimised Trench MOSFET Technologies for Power Devices”, IEEE Trans. Electr. Devices, vol.39, nº, Juin 1992. 